\title{
Storage Lifetime of Polymer-Carbon Nanotube Inks for Use as Charge Transport Layers in Organic Light Emitting Diodes
}

\author{
C. A. Mills ${ }^{1}$, F. L. M. Sam ${ }^{1}$, A. S. Alshammari ${ }^{1,2}$, L. J. Rozanski ${ }^{1}$, N. G. Emerson ${ }^{1}$ and S. R. P. Silva ${ }^{1}$ * \\ ${ }^{1}$ Advanced Technology Institute, University of Surrey, Guildford, Surrey, GU2 7XH, UK \\ ${ }^{2}$ Department of Physics, College of Science, University of Hail, P. O. Box 2440, Hail, Saudi Arabia \\ *Corresponding author: s.silva@surrey.ac.uk
}

\begin{abstract}
The long-term stability of multi-wall carbon nanotubes (MWCNT) mixed with the hole-transport polymer Poly(3,4ethylene dioxythiophene):poly(styrene sulfonate) (PEDOT:PSS) has been examined. These surfactant stabilised solutions, used as transport layers in organic light emitting diodes (OLEDs), are shown to be stable for periods of up to fifteen months, and show no signs of degrading soon after this time. In comparison, non-stabilised aqueous MWCNT solutions have been shown to aggregate within 30 minutes of production, and, although these aggregates can be re-dispersed, the solution displays an increase in smaller aggregates over time which cannot subsequently be re-dispersed by manual agitation. The stable MWCNT/PEDOT:PSS solutions have been used in ink-jet printing and as composite MWCNT/PEDOT:PSS films suitable as charge transport layers in spin coated organic light emitting diodes.
\end{abstract}

Keywords: Carbon nanotubes, Conducting materials, Organic Light Emitting Diodes, Polymer films 


\section{Introduction}

Organic electronics is a rapidly maturing technology with many commercial applications. Within the organic electronics field, Organic Light Emitting Diodes (OLEDs) show promise for the next generation lighting and display applications. Small area and pixelated OLEDs for lighting, display and television are already commercially available [1] and extensive research is on-going to develop large area OLED technology for lighting applications. [2] However, opportunities still exist for improving current OLED technology to a competitive level, particularly for large area inexpensive lighting in empathy with environmentally responsible life cycles and lifestyles. Currently the performance parameters, such as efficiency and lifetime, of OLEDs cannot match their inorganic analogues, [3] but the technology has suitable scope for improvement that future developments may well close the performance gap and give added flexibility and applications due to its solution processable form factors.

Although OLEDs offer a number of advantages over conventional inorganic-based technology, areas in which OLEDs could be improved have been identified during the development of the technology. [3] One area of significant interest that has been studied includes the interface between the light-emitting "emissive" material and the electrode "metal". Any mismatch between the energy levels in the organic active layer and the electrodes causes a reduction of the charge transport efficiency and device output as a result of the electrode/active layer interface. This in turn increases the required operating voltage, which strains the OLED active material and consequently reduces the lifetime of the OLED in addition to the wasted output due to the non-radiative recombination pathways.

One method to tackle this problem is by introducing interfacial layers between the electrodes and the active materials which produce intermediate energy states. These intermediate states reduce the energy discontinuity to transport charge carriers across interfaces, while decreasing the operating voltage and consequently extending the lifetime of the OLED. Here we present methods for the solution processing of multi-wall carbon nanotubes

(MWCNTS) for OLED applications. We report on the production and deposition of MWCNTs in Poly(3,4ethylenedioxythiophene) poly(styrenesulfonate) (PEDOT:PSS), a conducting polymer commonly used in organic electronic applications, and examine the lifetime stability of these solutions. 
We have previously reported on the use of MWCNTs as a hole transport layer to improve the device properties of OLEDs. [4] Using an Indium tin oxide (ITO) / MWCNTs / N, N'-bis(3-methylphenyl)-N,N'-diphenyl-1,1'-biphenyl-4,4'diamine (TPD) / Tris(8-hydroxyquinolinato) aluminium (Alq3) / Lithium Flouride (LiF) / Aluminium (Al) OLED, integrating a solution processed carbon nanotube layer, we reported a luminance approaching $50,000 \mathrm{~cd} \mathrm{~m}^{-2}$ with an external quantum efficiency over $2 \%$ and a current efficiency greater than $21 \mathrm{~cd} \mathrm{~A}^{-1}$ at an operating voltage of only 10V. Similarly, the integration of MWCNTs in PEDOT:PSS, in an ITO/PEDOT:PSS: 0.4 wt\% MWCNTS/TPD/Alq3/Al OLED, has been shown to lower turn-on voltages (approx. $10.5 \mathrm{~V}$ vs. $13.5 \mathrm{~V}$, defined as the voltage at $\left.500 \mathrm{~cd} \mathrm{~m}^{-2}\right)$ and obtain comparable luminescence values at lower operating voltages $\left(5000 \mathrm{~cd} \mathrm{~m}^{-2}\right.$ at approx. $14 \mathrm{~V}$ vs. $18 \mathrm{~V}$ ) than a reference ITO/PEDOT:PSS/TPD/Alq3/Al device. [5]

Such materials developed as the interface layers for OLEDs may also be used in alternative organic electronics applications. For example, MWCNT/PEDOT:PSS has been used in transistor devices as source/drain (S/D) electrodes. [6] A pentacene thin film transistor incorporating MWNT/PEDOT:PSS S/D electrodes displayed an increased mobility and on/off ratio when compared to pure PEDOT:PSS S/D electrodes: approx. $0.2 \mathrm{~cm}^{2} .(\mathrm{V} \mathrm{s})^{-1}$ and $5 \times 10^{5}$ compared to approx. $0.05 \mathrm{~cm}^{2} .(\mathrm{V} \mathrm{s})^{-1}$ and $1 \times 10^{5}$. The work function and sheet resistance of the MWCNT/PEDOT:PSS film has also been shown to be tuned by varying the weight percentage of MWCNTs in the PEDOT:PSS. Similarly, thin films of MWCNTs have been used to produce a low voltage rewritable memory device. [7] The ITO/PEDOT:PSS (MWCNT)/AI device displayed current bistability with a low resistance ON-state and high resistance OFF states remaining stable for hundreds of write-read-erase-read cycles over two hours.

The potential for organic materials to be deposited by large area, solution processable techniques such as ink-jet printing, roll-to-roll printing or spray coating is beginning to be realised. Yet, much development still needs to be performed before such a technology is adopted for production on a large scale. Consequently composite inks, such as MWCNT/PEDOT:PSS, may be required and if adopted will need to be stable for many months before utilisation in a device configuration. Here we examine the long term stability of MWCNT/PEDOT:PSS solutions over a period of fifteen months. 


\section{Methods}

2.1 Materials

\subsubsection{Chemicals}

Chemicals were used as received from the supplier. PEDOT:PSS (1.3-1.7 wt \% dispersion in $\mathrm{H}_{2} \mathrm{O}$, Clevios PVP AI4083 or $\mathrm{P}$ Jet conductive grade), was purchased from Heraeus. MWCNTs (carbon $>95 \%$, Outside diameter $=6-9 \mathrm{~nm}$, Length $=5 \mu \mathrm{m}$ prior to functionalization), Toluene (99.99\%) and reagent grade methanol (MeOH) and ethanol (EtOH) were purchased from Sigma-Aldrich Chemical Co., UK. Pre-sized (approx. $25 \times 25 \mathrm{~mm}^{2}$ ) and patterned indium tin oxide (ITO) coated glass (LT-G001, nominal sheet resistance $=9-15 \Omega \square^{-1}$, ITO thickness $=120-160 \mathrm{~nm}$, transparency $>84 \%$ [at 550nm]) was purchased from Luminescence Technology Corporation (Taiwan). Poly[2methoxy-5-(2-ethylhexyloxy)-1,4-phenylene-vinylene] (MEH-PPV, ADS100RE) was sourced from American Dye Source, Canada.

\subsubsection{MWCNT solution preparation}

Acid functionalization was completed using a previously described method. [4] Solutions of acid-functionalised MWCNTs have been prepared in water (0.5 wt\% MWCNT) and in aqueous PEDOT-PSS solution (0.625 wt\% MWCNT) incorporating surfactant $(0.2-1.0 \mathrm{wt} \%$, sorbitol or $1.0 \mathrm{wt} \%$ sodium dodecyl sulphate [SDS]). For the duration of the solution lifetime experiments, the solutions were simply stored on a shelf. No shaking or sonication of the solutions was undertaken between measurements, except in the case of the aqueous MWCNT solution which was shaken manually immediately prior to measuring the transmittance of the solution.

\subsection{Film preparation}

MWCNT/PEDOT:PSS films were deposited by spin coating and ink-jet printing. For spin coating, ITO was first rinsed with deionised water, then cleaned with successive 5 minute sonic baths of deionised water, methanol and ethanol. The ITO was then dried with nitrogen before exposure to an oxygen plasma (5 min, $100 \mathrm{~W}, 20 \mathrm{mbar} \mathrm{O}_{2}$, Emitec K1050X plasma cleaner). MWCNT/PEDOT:PSS solution was spin-cast (Laurell Technologies Corporation, 
USA) onto the ITO at 5000 rpm for $60 \mathrm{~s}$, giving a polymer film thickness of approx. $50 \mathrm{~nm}$. The MWCNT/PEDOT:PSS film was then annealed at $150{ }^{\circ} \mathrm{C}$ under a nitrogen atmosphere for 15 min to eliminate any trapped solvent/surfactant. As required, Al (100 nm thick) was thermally evaporated onto the PEDOT:PSS through a shadow mask, at a pressure of $5 \times 10^{-6}$ mbar.

For ink-jet printing, 0.03 wt\% of surfactant-dispersed MWCNT was mixed with the conductive PEDOT:PSS ink and sonicated for 30 minutes to ensure a homogeneous mixture. A syringe fitted with a syringe filter $(0.2 \mu \mathrm{m})$ was used to fill the print cartridges. The viscosity of the composite ink was measured using Brookfield cone-plate viscometer (at a speed of $100 \mathrm{rpm}$ and at room temperature) and found to be 12.8 centipoise (cP). The MWCNT/PEDOT:PSS conductive layers were printed using a Dimatix DMP-2831 printer with $10 \mathrm{pL}$ piezoelectric print heads. The jetting of the droplet was controlled by the firing voltage and the waveform which were optimized in order to achieve stable jetting of the droplets and produce high quality printed layers. The samples were printed at room temperature using a jetting voltage of $21 \mathrm{~V}$, drop spacing of $20 \mu \mathrm{m}$ and jetting frequency of $5 \mathrm{KHz}$. The distance between the print head and substrate was maintained at $1 \mathrm{~mm}$ during the printing of the samples. The ink-jet printed films were then imaged using scanning electron microscopy (SEM, Quanta 200, FEI, USA).

\subsection{OLED preparation}

OLEDs were fabricated on pre-patterned ITO substrates which were cleaned by immersion sequentially in acetone, isopropanol and methanol in an ultrasonic bath for 5 min each, followed by 5 min in a plasma asher (KM1050X Plasma Etcher/Asher/Cleaner from Quorum Technologies). A MWCNTs / PEDOT:PSS hole transport layer spin cast from a MWCNT / aq. PEDOT:PSS (sorbitol) solution (0.0625 wt\% MWCNT, 0.13-0.17 vol\% PEDOT:PSS, 1.0 wt\% sorbitol) at $2000 \mathrm{rpm}$ for $1 \mathrm{~min}$, then annealed at $150{ }^{\circ} \mathrm{C}$ for $10 \mathrm{~min}$. A second, planarising PEDOT:PSS layer (without CNTs) was spun down on the first at $2000 \mathrm{rpm}$ for $1 \mathrm{~min}$. Subsequent steps were performed in a nitrogen atmosphere, free of oxygen and water, inside a glove box. After spin-coating PEDOT:PSS, the substrate was annealed at $150{ }^{\circ} \mathrm{C}$ for $10 \mathrm{~min}$. under nitrogen. After cooling, MEH-PPV was deposited by spin coating from chloroform solution (5 mg.ml ${ }^{-1}$ ) for $1 \mathrm{~min}$. at $1000 \mathrm{rpm}$, before annealing at $150{ }^{\circ} \mathrm{C}$ for $10 \mathrm{~min}$. Subsequently, $2,9-$ dimethyl-4,7-diphenyl-1,10-phenanthroline (Bathocuproine, or BCP, $5 \mathrm{~nm}$ ) followed by Al (100 nm) were 
evaporated onto the MEH-PPV using a mask to define the OLED area. The device was encapsulated before characterisation, and stored under nitrogen in the dark.

\subsection{Characterization}

\subsubsection{UV-visible spectroscopy}

For the solution lifetime measurements, UV-visible transmission spectra (Camspec M330) have been recorded from 220 to $900 \mathrm{~nm}$ on solutions of MWCNT/PEDOT:PSS and aqueous MWCNTs, and compared with that for PEDOT:PSS only. In each case, the spectra were corrected for solvent/cuvette background signal. Temporal measurements were recorded at a single transmission wavelength $(420 \mathrm{~nm})$, using either computer acquisition (short time periods up to 1 hour) or manually (long time periods up to 15 months).

\subsubsection{Sheet resistance}

Sheet resistance is measured using the transfer length measurement (TLM) technique (also known as a transfer line measurement). [8] Al contacts are evaporated through a shadow mask onto two thin films of the sample under test. The Al contacts have dimensions of $0.1 \mathrm{~cm} \times 2.54 \mathrm{~cm}$ (corresponding to the width of the sample substrate) and are spaced to different distances $(1,3$, and $8 \mathrm{~mm}$ or 2,4 , and $6 \mathrm{~mm})$. Note that the width and length of the contacts are defined according to the direction of current conduction, and that the width is not necessarily shorter than the length, as:

$$
R_{T}=\frac{\rho l}{A}=\frac{\rho l}{w t}
$$

When,

$$
\begin{aligned}
& R_{s}=\frac{\rho}{t} \\
& R_{T}=R_{s} \frac{l}{w}
\end{aligned}
$$


where $R_{T}$ and $R_{s}$ are the total resistance and sheet resistance respectively, and $\rho$ is the resistivity. A, I, w and $t$ define the dimensions of the resistor (cross-sectional area, length [contact separation], width [contact width] and thickness respectively).

The sheet resistance can be calculated using equation $3 . \mathrm{R}_{\mathrm{T}}$ is measured between contacts that are separated by distance $\mathrm{I}$, and plotted. The slope of the graph is then equal to $R_{s} / w$, and $R_{s}$ can be calculated. Further parameters can be defined, such as the contact resistance, $R_{c}=\left(y\right.$-intercept/2), and the transfer length, $T_{L}=(x$-intercept/2).

\section{Results and Discussion}

Fig. 1 shows the chemical structures of PEDOT:PSS and the surfactants. The surfactant used, sorbitol or sodium dodecyl sulphate in this case, acts to help disperse the CNTs in the aqueous polymer solution. Fig. 1 also shows the UV-visible absorption spectra of the solutions used in the measurements: water diluted PEDOT:PSS solution, and MWCNTs suspended in water and in PEDOT:PSS. The aqueous MWCNT sample produces little in the way of spectral information, merely displaying an increase in absorption as the incident radiation moves to lower wavelengths. The PEDOT:PSS reference solution displays a characteristic peak at approx. $800 \mathrm{~nm}$.

Compared with the PEDOT:PSS reference, the peak at approx. $800 \mathrm{~nm}$ can also be seen in the MWCNT/PEDOT:PSS sample, although it is somewhat less prominent, possibly from dilution effects due to the addition of the MWCNTs and the surfactant. Alternatively, the interaction between the electronic structures of the PEDOT:PSS and the MWCNTs may reduce the PEDOT:PSS absorption at higher wavelengths. The MWCNT/PEDOT:PSS spectrum also displays the increase in absorption due to the MWCNTs, at wavelengths below the $800 \mathrm{~nm}$ PEDOT:PSS peak.

To be applicable for long term use, the solutions have been examined for their storage longevity. The use of high mass MWCNTs, when compared to single wall CNTs (SWCNTs), should amplify any settling of the CNTs out of solution. The diluted aqueous MWCNT solution has been found to aggregate with time, causing a consequent drop in the quantity of light absorbed by the sample. The use of the aqueous MWCNT solution (as opposed to a nonstabilised PEDOT:PSS solution) allows the effects of the aggregation to be observed visually, while still being 
comparable to the surfactant stabilised MWCNT/PEDOT:PSS solution as the latter contains only a low concentration of PEDOT:PSS (<0.2 wt.\%).

Neither the PEDOT:PSS, nor the CNTs, return much spectral information in the visible region of the electromagnetic spectrum allowing us to compare the aqueous solution with the MWCNTs dispersed in PEDOT:PSS (For comparison see [9] and [10] respectively). Consequently, an arbitrary wavelength can be chosen with which to compare spectra over extended periods of time. To this end we have chosen $\lambda=420 \mathrm{~nm}$, which avoids the spectral peak due to the PEDOT:PSS at approx. $800 \mathrm{~nm}$ and cuvette effects at wavelengths below approx. $300 \mathrm{~nm}$.

Fig. 2a shows the evolution of the MWCNT aggregates over time (line A) for a freshly prepared solution of MWCNTs in water. For approximately the first 10 minutes, the CNTs remain relatively well dispersed in solution, although the transmittance increases by approx. 4\%. After 10 minutes, the appearance of large peaks in the plot suggests the growth of sizable aggregates in the solution. The dashed line in the plot approximates to the increasing maximum transmission of the solution, equating to an increase in transparency of the solution as the CNTs aggregate and large gaps appear between the aggregates. The peaks extending downwards from this line, indicative of periodic points of greater opacity, are due to the larger CNT aggregates passing in front of the detector. These effectively block the light from the spectrometer light source, causing the transmission to fall to low levels ( $\leq 10 \%$ transmission).

The CNTs can be re-dispersed simply by shaking the container and visually there is no difference in opacity from the freshly prepared solution. However, examination of the re-dispersed solution approx. one month after preparation (Fig. 2a, line B) shows that the transmission immediately after re-dispersion is more opaque: approx. $44 \%$ transmission compared to approx. $51 \%$ transmission for the freshly prepared solution, this difference is denoted by $\alpha$ in the figure. This suggests that, although visually the solution appears to re-disperse well, smaller aggregates of CNTs form in the solution and survive the re-dispersion by shaking.

A closer examination of the data shows further differences between the freshly prepared MWCNT solution and the same solution re-dispersed one month later. An examination of the standard deviation of the mean transmission, taken from the average of 500 sequential measurement points of the data in Fig. 2a, shows that the standard 
deviation increases as the aggregates in the solution grow with time. Although the month old solution contains small aggregates of CNTs, as evidenced by the decrease in transmission in Fig. 2a, the solution remains stable for longer. The appearance of larger aggregates, indicated by the increase in the standard deviation, occurs more slowly in the month old sample. Using an indicative standard deviation of $1 \%$, the freshly prepared solution reaches this level after 10 min., whereas the month old solution takes 20 min. (indicated by $\beta$ in Fig. 2a). The higher opacity of the month old sample (seen at early time intervals in Fig. 2a) suggests that small MWCNT aggregates remain in the solution even after shaking. The longer time for the larger aggregates to form in the month old sample may be due to the presence of these small aggregates that are more stable than the fully dispersed MWCNTs in the original solution. This would mean that the small aggregates remain as individual entities for longer, before eventually agglomerating into larger aggregates. The higher standard deviation in the transmittance for the month old aq. MWCNT solution may be reduced by using a more rigorous approach to redistribute the CNTs, such as sonication.

If the aq. MWCNT solution is stabilised with the addition of surfactant, this aggregation occurs less readily (Fig. $2 \mathrm{~b}$ ). The addition of increasing concentrations of sorbitol $(0.2,0.5$ and 1.0 wt\%) to the aq. MWCNT (0.625 wt\%) solution extends the stability of the solution from $10 \mathrm{~min}$ up to approx. $4 \mathrm{~h}$. For each sorbitol concentration, the MWCNT aggregation follows the same mechanism. An initial period of stability is seen, before an increase in solution transmission occurs and the transmission curve becomes increasingly noisy as large aggregates appear in the solution. Higher sorbitol concentrations are expected to further stabilise the solution.

Fig. 3a shows the stability of the solutions over a period of 15 months, and compares the opacity of the MWCNT/PEDOT:PSS solution over this time with a pure PEDOT:PSS solution. The opacity of the aqueous MWCNT solution is seen initially to remain stable $(52.24 \pm 2.53 \%)$, even though it has to be manually shaken between measurements. However, after the first week, the solution becomes increasingly opaque as time progresses as the MWCNT aggregates become increasingly entangled and insoluble. The MWCNT/PEDOT:PSS solution on the other hand, although somewhat more opaque than the PEDOT:PSS due to the addition of the MWCNTs, retains a constant opacity $(32.84 \pm 0.93 \%)$ for the duration of the experiment, suggesting that the MWCNTs within remain fully dispersed in solution. In comparison, the pure PEDOT:PSS solution also retains a constant opacity (47.94 \pm 
$0.35 \%$ ). Shown inset in Fig. 3 are images of the freshly prepared polymer and the polymer/CNT solutions (Fig. 3b, see figure caption for key). Also shown are images of the aqueous MWCNT solution (0.5 wt\% MWCNT), after standing for 1.5 hours, showing the MWCNT aggregates formed, and immediately after manual shaking, showing the visually uniform colour of the re-dispersed solution (Fig. 3c). The stability of the MWCNT/PEDOT:PSS solution suggests that the charged macromolecules in the PEDOT:PSS add to the stabilisation effects of the sorbitol surfactant on the MWCNTs. As a polymer mixture of two ionomers, the negatively charged sodium polystyrene sulfonate and the positively charged poly(3,4-ethylenedioxythiophene), PEDOT:PSS acts as a macromolecular salt and electrostatically stabilises the MWCNTs.

The effect of the presence of surfactant in the PEDOT:PSS can be seen in Fig. 4. Increasing the sorbitol concentration decreases the sheet resistance by three orders of magnitude, from approx. $3 \times 10^{7}$ to $2 \times 10^{4} \Omega \square^{-1}$. The reason for this is that the surfactant causes an increased alignment of the PEDOT chains into larger PEDOT grains, and reduces the amount of PSS between these grains. [11] The surfactant is not found to affect the conductivity of the final PEDOT film as it evaporates during drying. Although a significant improvement is seen compared to the PEDOT:PSS without surfactant, the decrease in sheet resistance for the PEDOT:PSS films produced here does not yet rival the typical sheet resistance for the ITO-coated glass $\left(10-30 \Omega \square^{-1}\right)$ commonly used in optoelectronic applications, such as OLED production. The reduction in sheet resistance is however in line with that seen for MWCNTs dispersed in PEDOT:PSS, where the sheet resistance decreases from $10^{6}$ to $10^{3} \Omega \square^{-1}$. [6]

Finally, as an example of some applications for the MWCNT inks, we present the preparation of ink-jet printed MWCNT/PEDOT:PSS films, [12] and the incorporation of a spin coated MWCNT/PEDOT:PSS hole transport layer into an MEH-PPV OLED. Fig. 5 shows a MWCNT/PEDOT:PSS film deposited using ink-jet printing with a sheet resistance of $1.26 \times 10^{4} \Omega \square^{-1}$. The film is composed of multiple lines of MWCNT/PEDOT:PSS deposited on a paper substrate. The inset, magnified SEM image shows the smooth PEDOT:PSS film surface with well-spaced, single MWCNTs protruding from the film and lying on the surface of the PEDOT:PSS. The sheet resistance of the MWCNT/PEDOT:PSS film is comparable to the PEDOT:PSS films presented in Fig. 4, but in this case the added sorbitol concentration is lower (1 wt\% SDS) demonstrating that the lower resistance in this case is due to the inclusion of the MWCNTs. 
Also presented inset in Fig. 6 is an image of a pixelated ITO/PEDOT:PSS (MWCNT)/MEH-PPV/BCP/Al OLED with one of the OLED pixels illuminated. The OLED incorporates a spin coated MWCNT/PEDOT:PSS hole transport layer, produced using the diluted 15 month old MWCNT/PEDOT:PSS solution. Luminance and current density measurements are shown in figure 6. A comparative ITO/PEDOT:PSS/MEH-PPV/BCP/Al reference diode has a turn on voltage of $4.6 \mathrm{~V}$ and achieves a luminance of $1970 \mathrm{~cd} \cdot \mathrm{m}^{-2}$ at $9 \mathrm{~V}$. In comparison, the ITO/PEDOT:PSS(MWCNT)/MEH-PPV/BCP/AI OLED turns on at $5.2 \mathrm{~V}$ and achieves a luminance of $430 \mathrm{~cd} . \mathrm{m}^{-2}$ at $10 \mathrm{~V}$, before displaying breakdown character. The emission spectrum of the reference OLED has a peak maximum at 570 $\mathrm{nm}$. The ability to spin coat or ink-jet print these conducting MWCNT/PEDOT:PSS composite inks, allied to their proven shelf life, make them attractive for commercial applications.

\section{Conclusion}

The stability of solutions of MWCNTS/PEDOT:PSS has been determined for periods of up to fifteen months. Comparison of the opacity of a non-stabilized aqueous MWCNT solution to that of the surfactant-stabilized MWCNT/PEDOT:PSS solution shows that without the presence of the surfactant, the MWCNTs very quickly (in less than 30 minutes) aggregate out of solution. In contrast, the surfactant stabilized MWCNT/PEDOT:PSS solution is stable for up to fifteen months without the necessity for periodic re-dispersion or continuous agitation. This means that the stabilized MWCNT/PEDOT:PSS solution is useful for long term storage, and for use in applications such as ink-jet printing where the MWCNT/PEDOT:PSS ink solution may be required to stand for a long time between periods of use.

\section{Acknowledgments}

The authors thank Dr. D. Cox (Advanced Technology Institute, University of Surrey) for assistance with the production of the SEM images. This work was supported in part through an E.On Research Contract, the EPSRC KTA 
Published in: IEEE/OSA Journal of Display Technology

programme, and from the EU 7th Framework project, Smartonics, under grant agreement number 310229. A. A. acknowledges support from the University of Hail and the Saudi Arabia cultural bureau. 


\section{References}

1. B. Geffroy, P. le Roy, C. Prat (2006) “Organic light-emitting diode (OLED) technology: materials, devices and display technologies" Polym. Int. 55, 572-582

2. J. Park, J. Lee, D. Shin, S. Park (2009) "Luminance uniformity of large-area OLEDs with an auxiliary metal electrode" J. Disp. Technol. 5, 306-311

3. J. N. Bardsley (2004) “International OLED Technology Roadmap” IEEE J. Sel. Topics Quantum Electron 10, 3-9

4. S. Shi, S. R. P. Silva (2012) "High luminance organic light-emitting diodes with efficient multi-walled carbon nanotube hole injectors" Carbon 50, 4163-4170

5. G-F. Wang, X-M. Tao, R-X. Wang (2008) “Fabrication and characterization of OLEDs using PEDOT:PSS and MWCNT nanocomposites" Composites Science and Technology 68, 2837-2841

6. D-J. Yun, K. Hong, S. H. Kim, W-M. Yun, J-Y. Jang, W-S. Kwon, C-E. Park, S-W. Rhee (2011) "Multiwall Carbon Nanotube and Poly (3, 4-ethylenedioxythiophene): Polystyrene Sulfonate (PEDOT: PSS) Composite Films for Transistor and Inverter Devices" ACS Appl. Mater. Interf. 3, 43-49

7. J. A. Ávila-Niño, W. S. Machado, A. O. Sustaita, E. Segura-Cardenas, M. Reyes-Reyes, R. López-Sandoval, I. A. Hümmelgen (2012) “Organic low voltage rewritable memory device based on PEDOT:PSS/f-MWCNTs thin film” Organic Electronics 13, 2582-2588

8. G. K. Reeves, H.B. Harrison (1982) "Obtaining the specific contact resistance from transmission line model measurements" IEEE Electron. Device Lett. 3, 111-113

9. K. S. Kang, H. K. Lim, K. Y. Cho, K. J. Han, J. Kim (2008) “Durability of PEDOT : PSS-pentacene Schottky diode” J. Phys. D: Appl. Phys. 41, 012003

10. P. Yadav, R. Ajore, L. M. Bharadwaj (2009) “Cross-Linker Mediated Biofunctionalization of Single Wall Carbon Nanotubes with Glucose Oxidase" J. Nanotechnol. Online, 5, DOI: 10.2240/azojono0130

11. J. Huang, P. F. Miller, J. S. Wilson, A. J. de Mello, J. C. de Mello, D. D. C. Bradley (2005) "Investigation of the effects of doping and post-deposition treatments on the conductivity, morphology, and work function of Poly(3,4-ethylenedioxythiophere)/Poly(stryrene sulfonate) films" Adv. Funct. Mater. 15, 290-296 
Published in: IEEE/OSA Journal of Display Technology

12. A. S. Alshammari, M. Shkunov, S. R. P. Silva "Effect of carbon nanotube alignment on the properties of ink-jet printed PEDOT:PSS/MWCNT Nano-composites" in preparation 
Figure captions

Fig 1. UV-visible spectra of (O) MWCNT / H2O (0.5 wt\% MWCNT), (O) aq. PEDOT:PSS (0.13-0.17 vol\% PEDOT:PSS) and ( $\boldsymbol{\Delta})$ MWCNT / aq. PEDOT:PSS (sorbitol) (0.625 wt\% MWCNT, 0.13-0.17 vol\% PEDOT:PSS, 1.0 wt\% sorbitol). Inset are the chemical formulae for (a) PEDOT:PSS, (b) sorbitol, and (c) sodium dodecyl sulphate respectively.

Fig 2. (a) Extent of aggregation of MWCNTs (0.5 wt.\%) in water over approx. 1h, monitored by recording the transmittance (\%) of the solution at $420 \mathrm{~nm}$ with respect a water reference. The data are recorded (A) immediately after solution preparation and (B) after 1 month shelf storage. The dashed line in each case approximates to the maximum transmission of the solution with time. (b) Extent of aggregation of MWCNTs (0.625 wt\%) in water with time, monitored by recording the transmittance (\%) of the solution at $420 \mathrm{~nm}$ with respect a water reference. The solutions contain (A) 0 (reproduced from Fig. $2 a$ to the point that major fluctuations occur due to the presence of aggregates), (B) $0.2,(C) 0.5$, and (D) 1.0 wt.\% sorbitol surfactant. Data are normalised for comparison.

Fig 3. (a) Long duration comparison of the transmission of solutions of (O) MWCNT / H2O (0.5 wt\% MWCNT), (O) aq. PEDOT:PSS (0.13-0.17 vol\% PEDOT:PSS) and ( $\mathbf{\Delta}$ ) MWCNT / aq. PEDOT:PSS (sorbitol) (0.625 wt\% MWCNT, 0.13-0.17 vol\% PEDOT:PSS, 1.0 wt\% sorbitol), monitored by recording the transmittance (\%) of the solution at $420 \mathrm{~nm}$ with respect a water reference. Solid lines are the average of the data points; the dashed line provides a guide for the eye only. (b) Images of the freshly prepared polymer and polymer/CNT solutions. Key (from I-r): 0. empty cuvette, 1. Water blank, 2. MWCNT / H2O (0.5 wt\% MWCNT), 3. PEDOT:PSS blank (0.13-0.17 vol\% PEDOT:PSS), 4. MWCNT / aq. PEDOT:PSS (sorbitol) (0.625 wt\% MWCNT, 0.13-0.17 vol\% PEDOT:PSS, 1.0 wt\% sorbitol). (Cuvette path length: $5 \mathrm{~mm}$ ). (c) Images of the MWCNT / H2O solution (0.5 wt\% MWCNT), (left) after standing for 1.5 hours and (right) immediately after manual shaking.

Fig 4. Sheet resistance, measured using the Transfer Length Measurement (TLM) technique, of PEDOT:PSS thin films $(50 \mathrm{~nm}$ ) deposited from solutions with increasing sorbitol concentration. The values highlighted by 
the black bar are the typical band of sheet resistances of ITO-coated glass used in current optoelectronics applications, such as OLED production.

Fig 5. SEM image of lines of PEDOT:PSS containing MWCNTs printed using ink-jet printing from a MWCNT / aq. PEDOT:PSS (SDS) solution (0.03 wt\% MWCNT, 0.13-0.17 vol\% PEDOT:PSS, 1.0 wt\% SDS) [bar = 1 mm]. Inset: (top right) Magnified image of the PEDOT:PSS film showing the incorporated MWCNTs [bar = 10 $\mu \mathrm{m}]$.

Fig 6. Luminance (closed symbols) and current density (open symbols) data for an ITO/PEDOT:PSS (MWCNT)/MEH-PPV/BCP/AI OLED produced using a hole transport layer spin cast from a MWCNT / aq. PEDOT:PSS (sorbitol) solution (0.0625 wt\% MWCNT, 0.13-0.17 vol\% PEDOT:PSS, 1.0 wt\% sorbitol) (triangles), and a reference ITO/PEDOT:PSS/MEH-PPV/BCP/AI OLED (circles). Inset, image of an illuminated pixel of the ITO/PEDOT:PSS (MWCNT)/MEH-PPV/BCP/AI OLED. (Note, the image of the OLED is cropped and contrast enhanced to show the detail) 
Figures

Figure 1

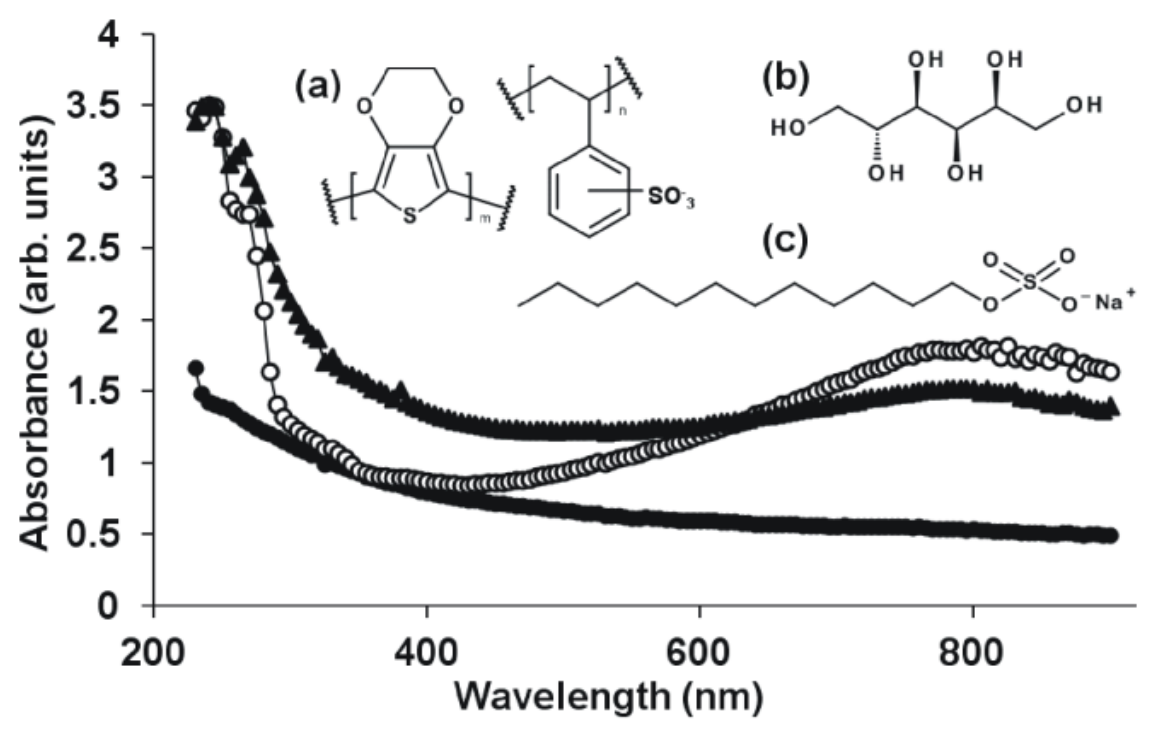

Figure 2

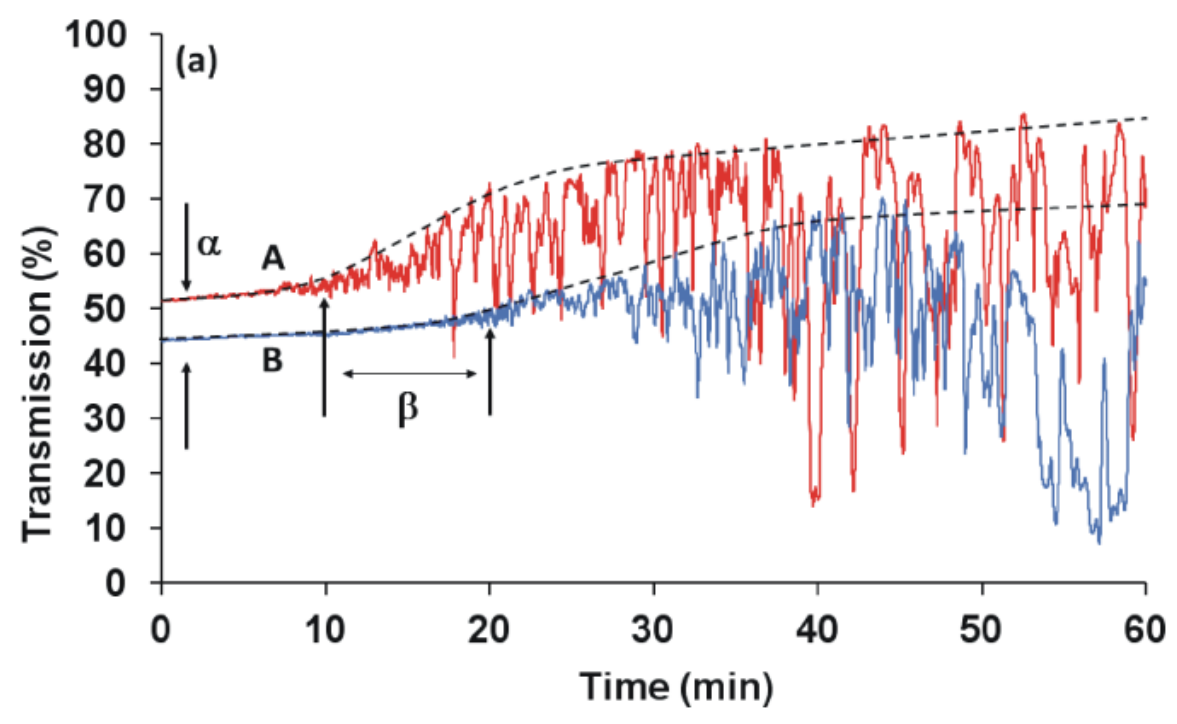




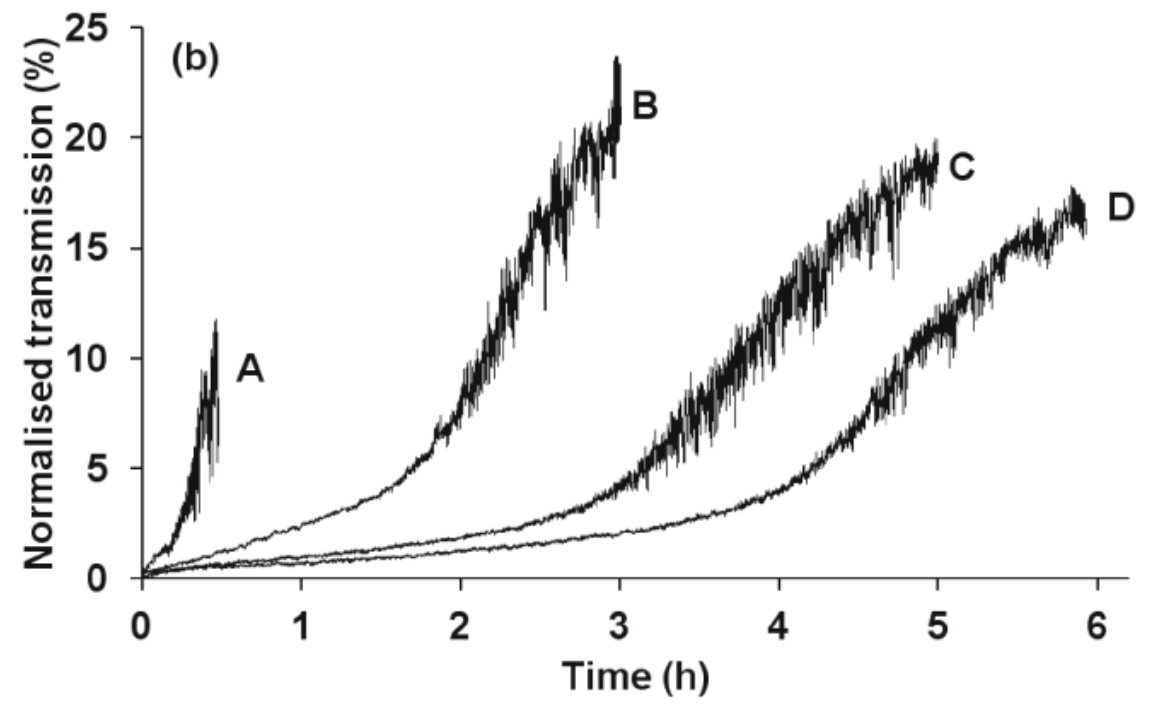

Figure 3

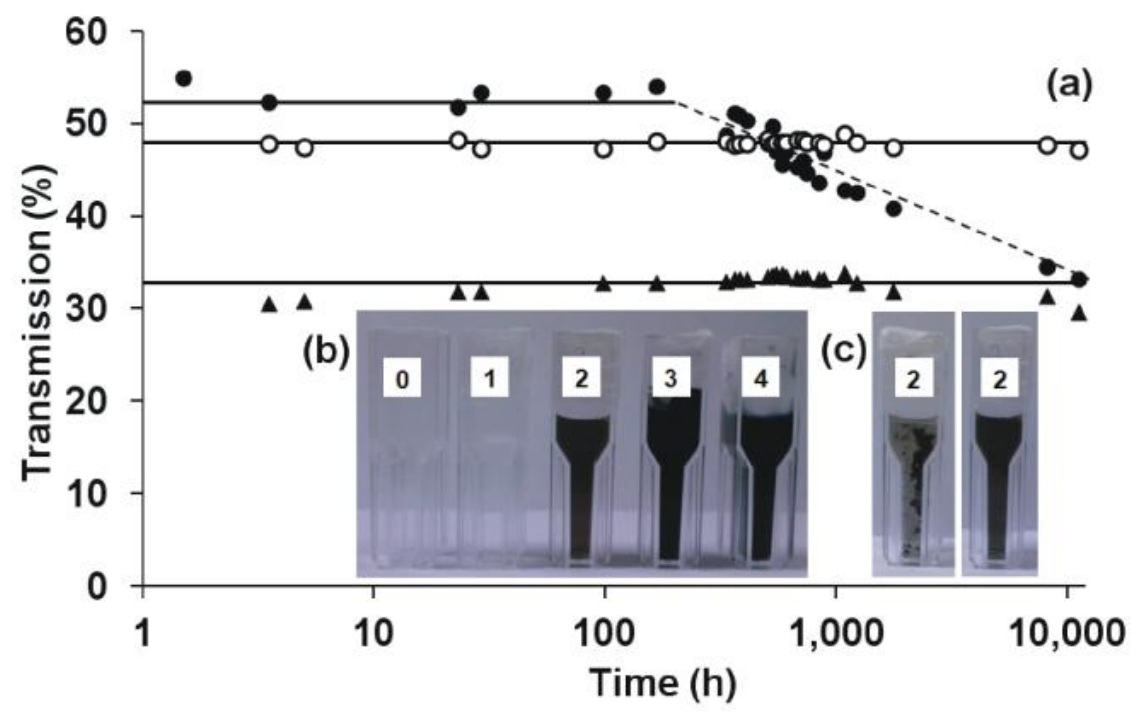


Figure 4

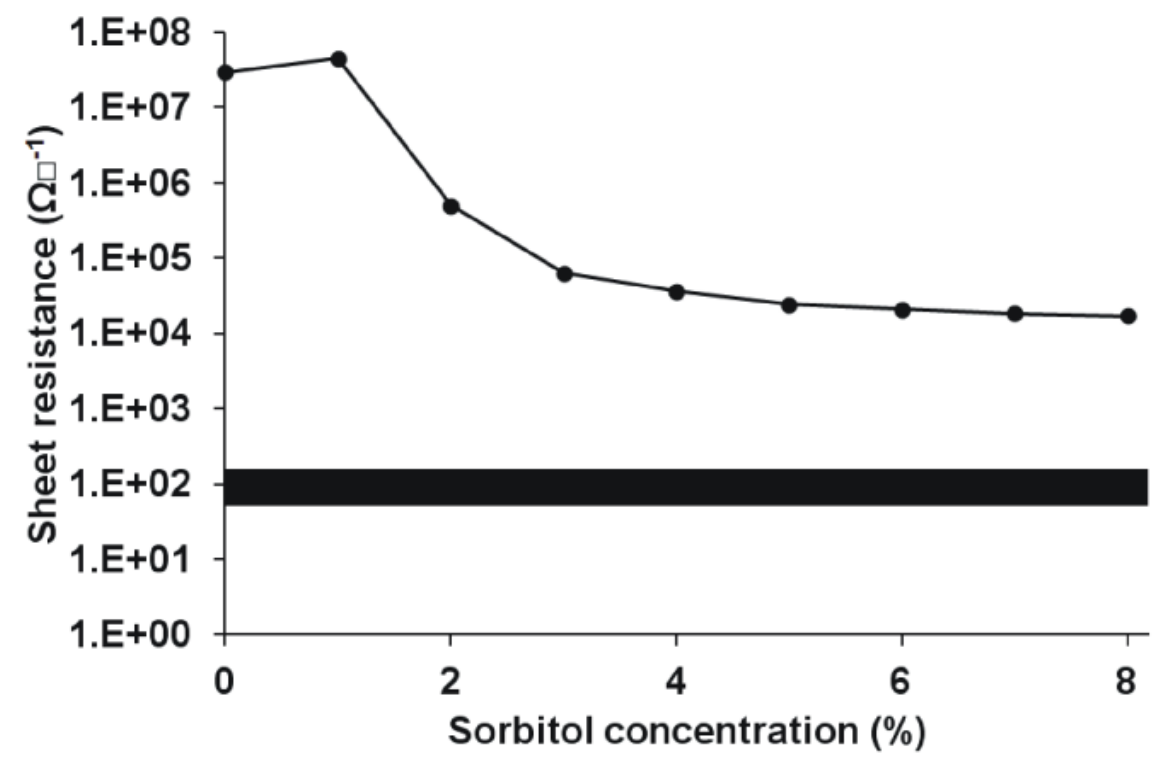

Figure 5

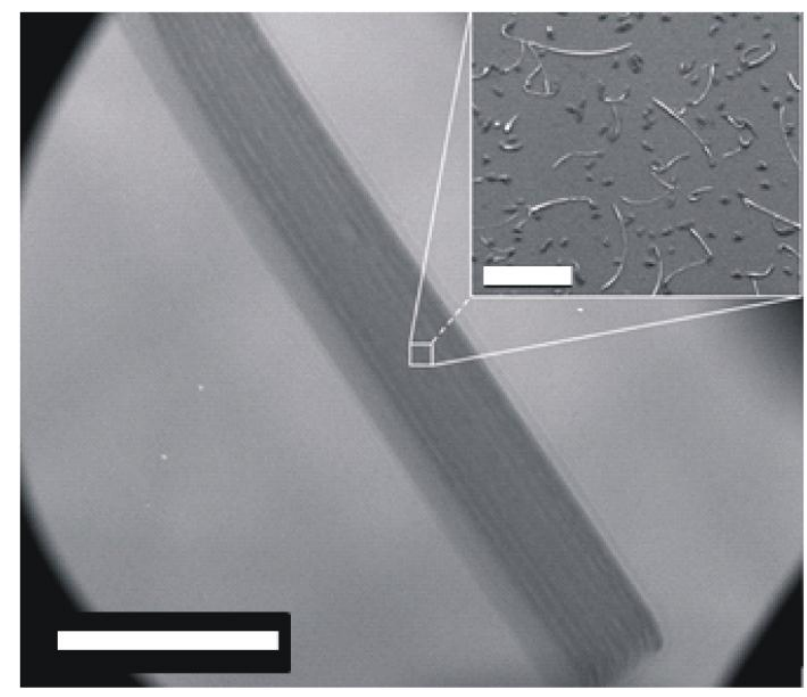


Figure 6

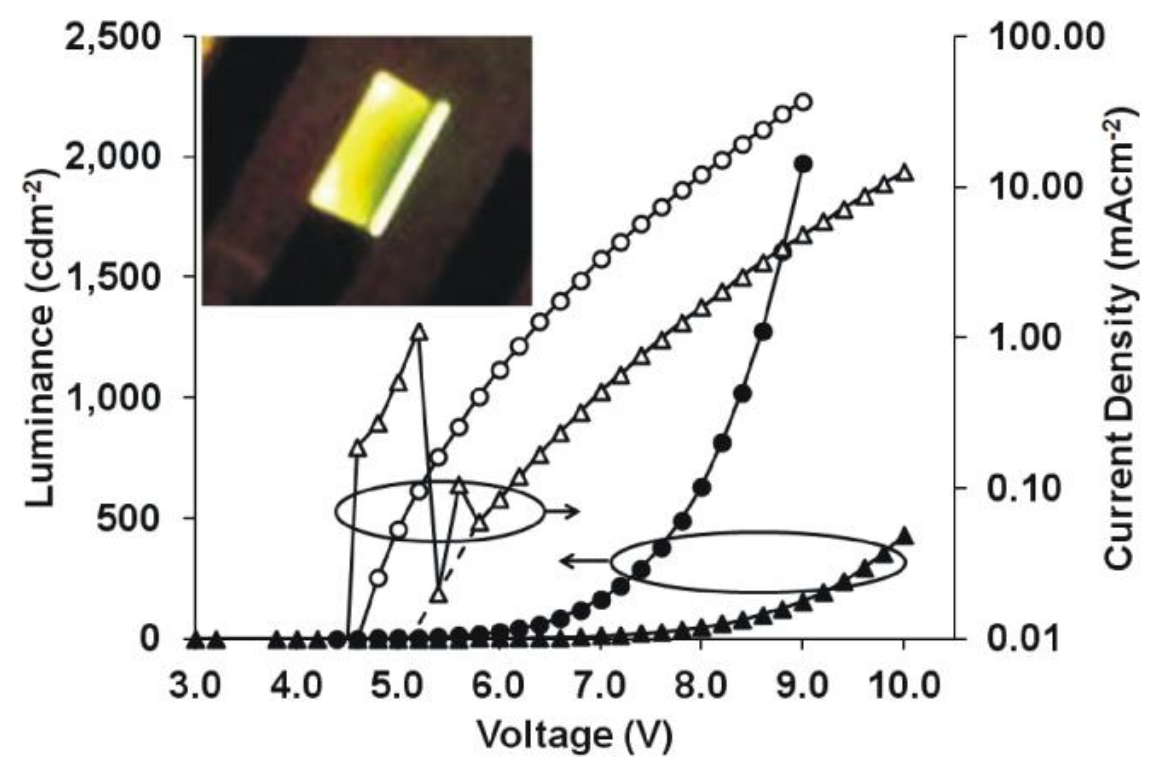

